\title{
Editorial
}

\section{Lattice and Magnetic and Electronic Transport Properties in Antiperovskite $M_{3} A X$ Compounds}

\author{
Cong Wang, ${ }^{1}$ Koshi Takenaka, ${ }^{2}$ Laifeng $\mathrm{Li}^{3}{ }^{3}$ and Yuping Sun ${ }^{4}$ \\ ${ }^{1}$ Department of Physics, Center for Condensed Matter and Material Physics, Beihang University, Beijing 100191, China \\ ${ }^{2}$ Department of Crystalline Materials Science, Nagoya University, Nagoya 464-8603, Japan \\ ${ }^{3}$ Technical Institute of Physics and Chemistry, Chinese Academy of Sciences, Zhongguancun East Road 29, Beijing 100190, China \\ ${ }^{4}$ Key Laboratory of Materials Physics, Institute of Solid State Physics, Hefei 230031, China
}

Correspondence should be addressed to Cong Wang; congwang@buaa.edu.cn

Received 29 January 2013; Accepted 29 January 2013

Copyright (C) 2013 Cong Wang et al. This is an open access article distributed under the Creative Commons Attribution License, which permits unrestricted use, distribution, and reproduction in any medium, provided the original work is properly cited.

In the past 50 years, perovskite $\mathrm{ABO}_{3}$ oxides have been extensively studied, specially in electronic transport for the example of high-Tc cuprate superconductors with aberrant perovskite structure and in correlation between spin ordering and electronic transport for the example of giant magnetoresistance $\mathrm{RMnO}_{3}$ (R: rare earth) compounds. However, another kind of compounds $\mathrm{M}_{3} \mathrm{AX}$ (M: magnetic elements, $\mathrm{Mn}, \mathrm{Ni}, \mathrm{Fe}$, etc.; A: transition and main group elements, $\mathrm{Ga}, \mathrm{Cu}, \mathrm{Sn}$, and $\mathrm{Zn} ; \mathrm{X}: \mathrm{N}, \mathrm{C}$, and $\mathrm{B}$ ), which was first discovered in 1930 by Morral as metallic perovskite, is lacking in systematical studies. The kind of compounds $M_{3} A X$, with the so-called antiperovskite structure due to the reverse occupancy of metal and nonmetal elements (such as $\mathrm{N}$ and C) in cubic body corner and body center positions, has also exhibited a wide range of interesting physical properties, such as superconductivity (SC), giant magnetoresistance (GMR), negative/zero thermal expansion (NTE or ZTE) and nearly zero temperature coefficient of resistivity (NZ-TCR), magnetostriction and piezomagnetic effects, and magnetocaloric effect (MCE) due to the strong correlation among lattice, spin, and charge. Therefore, these compounds have attracted great attention. Early reports suggested that the physical properties of these materials are mainly originated from the complex magnetostructure and the induced variable band structure. The strong coupling among crystal structure, magnetic, and even electric ordering parameters and/or fields is also of great interest for the design of novel magnetoelectronic devices. The ability to control and tune the electronic transport, magnetic transition, and abnormal thermal expansion behaviors of these materials makes it worthy to be studied for fundamental research as well as for potential applications.

Abnormal lattice variation has been one of the studied hot points in condensed matter physics. People have been searching for negative thermal expansion and nearly zero thermal expansion materials for many actual applications, which are very important in optical, microelectronic devices and spacecraft. Nowadays, the important phenomena were widely found in the series of antiperovskite compounds. However, the control of abnormal thermal expansion behaviors has been difficult, and how to design and obtain the nearly zero thermal expansion materials is still not clear. Materials with tunable magnetostructural correlations which may induce the lattice variation are very important. Such control over the magnetostructure and lattice variation has a great impact on material science.

This special issue deals with comprehensive contents in the field of condensed matter and materials physics, and it is interesting to the physicists and material researchers, who are studying on antiperovskite structured $\mathrm{M}_{3} \mathrm{AX}$ compounds or relevant functional materials. This special issue brings together some of the latest developments in this field of research. Many of them contain new and original results which we acknowledge with thanks.

The seven articles in the special issue can address but a small subset of these fields. P. Tong and Y. P. Sun presented a review paper on the Ni-based antiperovskite compounds. 
In more detail, they reported the recent research progress on the experimental investigations of superconducting $\mathrm{MgCNi}_{3}$ single crystals and the synthesis and physical properties of the neighbor compounds of $\mathrm{MgCNi}_{3}$. Moreover, a universal phase diagram of these compounds is presented, which suggests a phonon-mediated mechanism for the observed superconductivity. We know that it is still not clear whether the mechanism for $\mathrm{MgCNi}_{3}$ superconductivity is conventional or not. The central issue is if and how the ferromagnetic spin fluctuations contribute to the cooper paring. The paper by S. Lin, B. S. Wang, P. Tong et al. reported the magnetic and electrical properties of $\mathrm{GaCFe}_{3-x} \mathrm{Cr}_{x}(0 \leq x \leq 0.9)$, and a magnetic/electrical phase diagram was plotted. Furthermore, the Fermi-liquid behavior was obtained below $70 \mathrm{~K}$ in the sample. In another paper, they theoretically investigated the antiperovskite chromium-based carbides $\mathrm{ACCr}_{3}$ by the first principles calculation based on density functional theory (DFT). Y. Sun et al. reported the magnetic, calorimetric, and electronic transport properties of $\mathrm{Mn}_{3} \mathrm{SbN}$. The lattice distortion from the high-temperature cubic structure to the roomtemperature tetragonal structure, which accompanies a ferrimagnetic transition, introduces a large transition entropy of $10.2 \mathrm{~J} \mathrm{~mol}^{-1} \mathrm{~K}^{-1}$ near $353 \mathrm{~K}$. Y. Yin et al. reported the magnetic and electronic transport properties of $\mathrm{Mn}_{3} \mathrm{Zn}_{0.9} \mathrm{Cu}_{0.1} \mathrm{~N}$. The absolute value of $d \rho / d T$ of $\mathrm{Mn}_{3} \mathrm{Zn}_{0.9} \mathrm{Cu}_{0.1} \mathrm{~N}$ is much lower which is close to zero. The paper by T. Yamazaki et al. reported that the micrometer-scaled ferromagnetic $\mathrm{ZnN}_{y} \mathrm{Ni}_{0.6} \mathrm{Co}_{2.4}$ domains are formed and embedded within a superconductive $\mathrm{ZnNNi}_{3}$ bulk, which shows a chemical phase separation of superconductive $\mathrm{ZnNNi}_{3}$ and ferromagnetic $\mathrm{ZnN}_{\gamma} \mathrm{Ni}_{0.6} \mathrm{Co}_{2.4}$, reflecting the existence of a miscibility gap in $\mathrm{ZnN}_{y} \mathrm{Ni}_{3-x} \mathrm{Co}_{x}$ for the samples with $x<2$. B. Y. Qu et al. reported the giant negative thermal expansion in the Ge-doped antiperovskite $\mathrm{Mn}_{3} \mathrm{CuN}$ compound, which was theoretically studied using the first principles calculations. The giant negative thermal expansion was attributed to a magnetic phase transition from $\Gamma_{5 \mathrm{~g}}$ phase to the PM phase. In detail, they thought that these donated conduction electrons polarize the local electrons and change the local magnetic moments of $\mathrm{Mn}$ ions, leading to the volume contraction of the compound.

\section{Acknowledgment}

We would like to thank all contributors for their very hard work and patience in bringing out this special issue. We also expect that the special issue will stimulate the continuing efforts to deepen the investigation on the antiperovskite $\mathrm{M}_{3} \mathrm{AX}$ compounds.

Cong Wang Koshi Takenaka

Laifeng $\mathrm{Li}$

Yuping Sun 

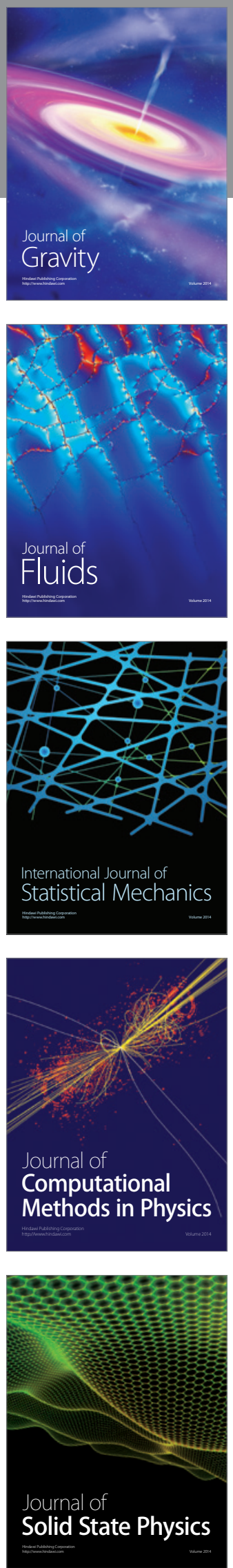

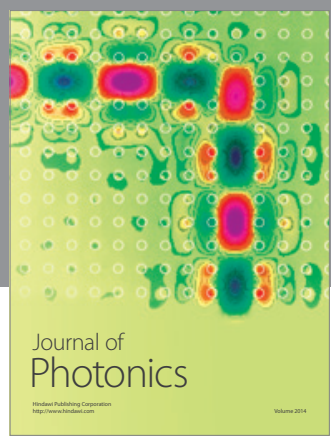

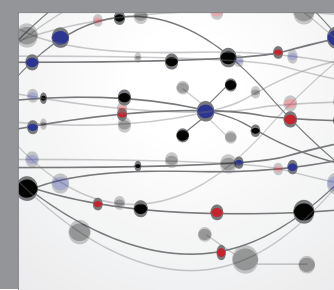

The Scientific World Journal

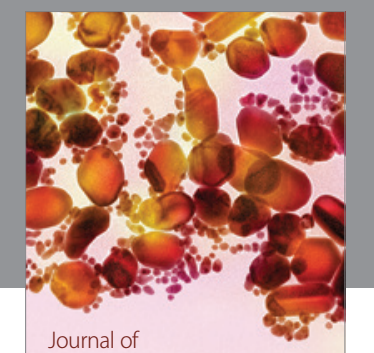

Soft Matter
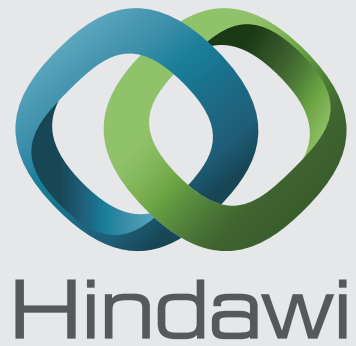

Submit your manuscripts at

http://www.hindawi.com
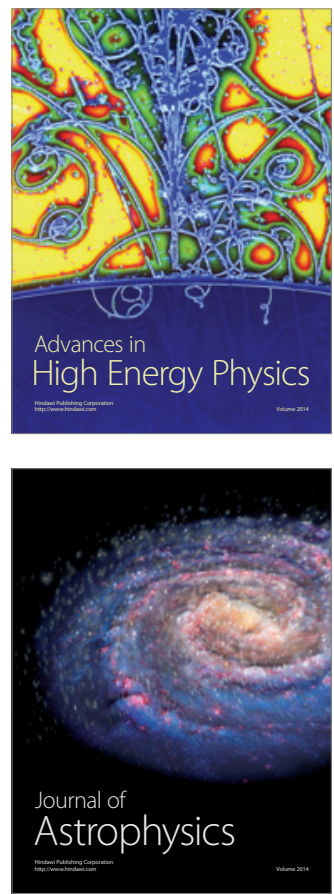
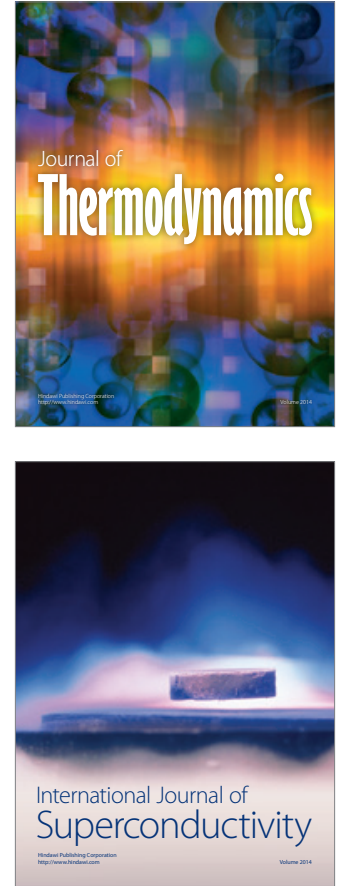
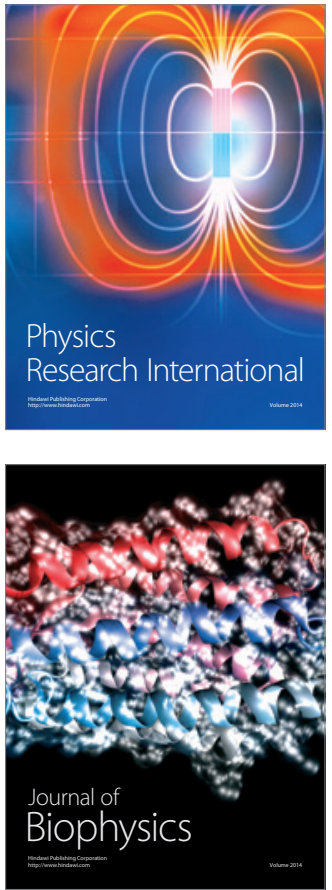
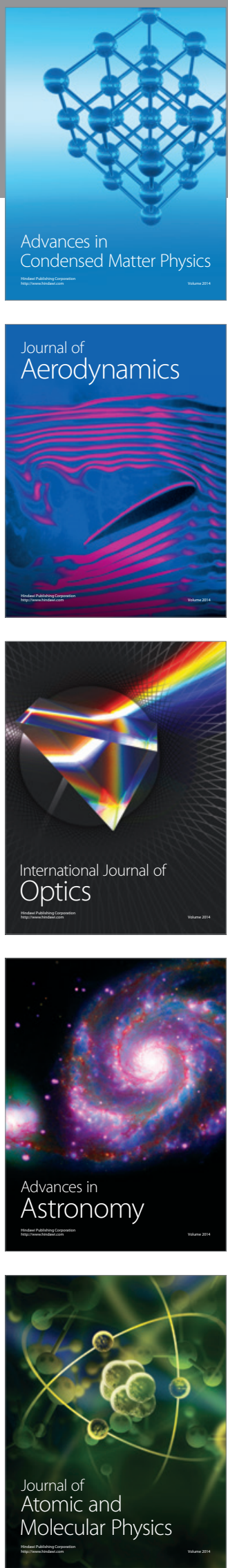